\title{
Reconfigurations existentielles chez Paul Tillich et Paul Ricoeur
}

\section{Elisabeth de Bourqueney*}

\section{Résumé}

Deux penseurs, à la frontière de la philosophie et de la théologie, Paul Tillich et Paul Ricoeur, ont affronté, et réinterprété des épreuves de leur existence, dans des textes de différentes natures, autobiographiques, philosophiques, systématiques. Paul Tillich a recours à la métaphore de la frontière et Ricœur parle du «vendredi saint « ou du samedi saint » pour évoquer deux événements différents.

Mots-clés : Situations-limites, expérience, métaphore, Vendredi-Saint, frontière.

\section{Paul Tillich and Paul Ricoeur Existential Reconfigurations}

\begin{abstract}
Two thinkers, at the border of philosophy and theology, Paul Tillich and Paul Ricoeur, faced, and reinterpreted the trials of their existence, in texts of different natures, autobiographical, philosophical, systematic. Paul Tillich uses the border metaphor and Ricœur speaks of "Good Friday" or of Holy Saturday "to evoke two different events. Keywords: Boundary situations, experience, metaphor, Holy Friday, border.
\end{abstract}

\section{Reconfigurações existenciais em Paul Tillich e Paul Ricoeur}

\section{Resumo}

Dois pensadores, na fronteira da filosofia e da teologia, Paul Tillich e Paul Ricoeur, enfrentaram e reinterpretaram as provações de sua existência, em textos de diferentes naturezas, autobiográficas, filosóficas, sistemáticas. Paul Tillich usa a metáfora da fronteira e Ricœur fala de "Sexta-feira Santa" ou do Sábado Santo "para evocar dois eventos diferentes.

Palavras-chave: Situações de fronteira, experiência, metáfora, Sexta-feira Santa, fronteira.

\footnotetext{
* Doctorat théologie protestante (Institut Protestant de Théologie Paris-Montpellier (2020), pasteur de l'Union des Eglises Protestantes, d'Alsace-Lorraine.

E-mail elsadebourqueney@orange.fr
} 
Le nuage pandémique qui traverse notre monde laissé exsangue nos corps, nous espérances et nos illusions. Où se trouve la transcendance dans nos existences bouleversées, menacées et à à redécouverte de leur fragilité? Nous avons été confrontés à une forme de néant relationnelle pour certains à un trop plein relationnel, laissant peu de place à l'intime.. A travers le choc inédit du confinement, nous avons expérimenté une situation-limite. Nous avons dû repenser notre place dans l'espace social, intime, spirituel. Nous avons commencé à reconfigurer de nouvelles relations, de nouvelles écritures et nous ne saisons pas encore toutes les formes et les implications de ces nouvelles formes d'écriture. Il serait bon de voir comment deux penseurs qui sont à la frontière de la philosophie et de la théologie, Paul Tillich et Paul Ricoeur, ont en leur temps, affronté, réinterprété et reconfiguré les épreuves de leurs existences, dans des textes de différentes natures, autobiographiques, philosophiques, systématiques. Il existe un lien entre pensée et expérience, lien dont on peut cherche les traces dans des textes de formes différentes, systématiques et autobiographiques, où la pensée apparait comme une forme de distanciation à l'égard d'expérience vécue et possède une proximité inavouée avec cette expérience. La fragilité apparait sous la métaphore de brisure, faille, fêlure, toutes choses qui confirment l'idée de rupture constitutive de la pensée sur la fragilité. Elles ne sont pas sans rappeler la métaphore de la croix. Quelles métaphores traversent leur œuvre en lien avec la fragilité, la passion et la résurrection ? Le travail métaphorique est oeuvre de reconfiguration qui permet une distanciation et une réappropriation pour l'auteur, qui peut être repris par un ou des lecteurs. Quelles translations de thèmes bibliques, quelles épreuves christiques sont reconfigurées par les philosophes de la religion? Paul Ricœur parle deux fois du « vendredi saint « ou du samedi saint » pour évoquer deux événements différents : la perte de son fils et l'expérience de captivité. Nous leur consacrerons deux moments. Quant à Paul Tillich, l'image de la frontière et de l'exil est récurrente. Comment les métaphores de la croix et de la frontière circulent-elles dans les écrits systématiques et peuvent-elles sous-tendre les récits et expressions autobiographiques et littéraires?

\section{Situations-limites et expressions- limites}

La notion de situation-limite a été initiée par le Philosophe Karl Jaspers. Dans son Introduction à la philosophie, Karl Jaspers, penseurfrontière, à la fois philosophe et psychiatre, affirme :« Il me faut mourir, il 
me faut souffrir, il me faut lutter ; je suis soumis au hasard, je me trouve pris inévitablement dans les lacets de la culpabilité. Ces situations fondamentales qu'implique notre vie, nous les appelons situations-limites. Cela veut dire que nous ne pouvons pas les dépasser, nous ne pouvons pas les transformer. En prendre conscience, c'est atteindre, après l'étonnement et le doute, l'origine plus profonde de la philosophie »( JASPERS, 2001 , p. 46-47). Face à ces situations-limites, l’homme peut réagir par la dissimulation ou « par le désespoir et une sorte de rétablissement : nous devenons nous-mêmes, par une métamorphose de notre conscience de l'être »(JASPERS, 2001, 48). Il y rencontre ce qui le transcende. Paul Tillich, quant à lui, considère le désespoir «comme une situation extrême ou une "situation-limite »; on ne peut aller au delà. Sa nature est suggérée par l'étymologie du terme désespoir : sans espoir. Aucune issue dans l'avenir n'apparaît. Le non-être est ressenti comme absolument victorieux, mais sa victoire est limitée » ( TILLICH, 1967, p. 43). A son tour, tandis qu'il étudie le thème biblique de la sagesse, Paul Ricœur reprend le terme de situations-limites que Karl Jaspers nomme "grenzsituationem » dans Psychologie der Weltanschauungen (JASPERS, 1954, p. 229 ). Ces situations limites sont relationnelles, dans leur rapport à soi, au monde et à autrui : "les thèmes de la sagesse sont les situations-limites où s'affrontent la grandeur et la misère de l'homme : à savoir la solitude, la faute, la souffrance et la mort. La sagesse hébraïque interprète ces situations comme néant de l'homme, incompréhensibilité de Dieu, voire silence et absence de Dieu »(RICOEUR, 2010, p. 218).

Face aux situations-limites de détresse, se dressent des expressionslimites. On trouve dans la Bible des expression-énigmes ou expressionslimites, telles que « royaume de Dieu « qui « mobilisent chez le lecteur des attentes opaques et muettes concernant la libération du mal (RICOEUR, 1982, p. 360 ). L'imagination s'exerce dans le travail du texte biblique et dans l'intertextualité, lorsque l'expérience du mal et de la souffrance est reconfiguré dans le récit. L'herméneutique relie expériences-limites et expressions limites. Des personnages sont rassemblées dans des paraboles, des récits " extravagants » qui témoignent du royaume de Dieu. "Il y a une correspondance entre les expressions-énigmes des paraboles et «des expressions semblables que l'évangéliste a mises en place dans le prologue qui précède le récit du ministère de Jésus et dans l'épilogue de la résurrection, - expressions par lesquelles le sens kérygmatique de tout l'Evangile est anticipé (Fils de Dieu, Seigneur, Christ)»(RICOEUR, 1982, p. 360). Paul 
Ricœur définit la métaphore comme une métamorphose en corrélation avec l'invitation à se déplacer, durer et devenir. l'expérience du mal est reconfiguré également par des métaphores christiques comme vendredi ou samedi saint. On ne peut échapper à l'épreuve mais par la relecture de l'événement intégré au récit sur sa propre histoire, ou par une image analogique, il est possible de reprendre le cours de sa vie.

\section{Paul Tillich Tillich et les chocs de son existence : une relecture de la frontière}

Paul Ricœur a rédigé la préface d'un ouvrage sur Paul Tillich et le symbole religieux en 1977 par Dimply. Pour lui, la corrélation est une « frontière franchie »( RICOEUR, 1977, p. 14). Dans la Théologie systématique, les corrélations sont multiples : ontologiques, existentielles, symboliques, spéculatives, culturelles. La Théologie systématique voit se déployer le réalisme symbolique de Tillich dans une lecture globale du sens de la préoccupation ultime : le symbole est « un mot creuset au double sens d'un lieu de mélange et d'un lieu d'épreuve » (RICOEUR, 1977, p. 11-12). Le concept de frontière est choisi comme emblématique de sa pensée, comme «cellule et matrice d'un ensemble métaphorique et discursif qui pourrait bien soutenir la relation de corrélation elle-même. La corrélation n'est-elle pas toujours une frontière franchie » (RICOEUR, 1977, p. 14).

On peut voir la frontière comme une métaphore centrale repensée à partir de l'expérience de l'exil. Tillich évoque des formes différentes de frontières, comme autant de métaphores, d’images. Ainsi, il parle de la raison face aux murailles de la finitude, des fêlures existentielles face aux ambiguïtés de la vie ou encore des fossés. Certains sont infranchissables, d'autres lignes sont déplacées. Tillich n'a eu de cesse de rechercher des concepts aux frontières de la religion, la culture, la science et en même temps de remettre en cause d'autres frontières, entre Dieu et l'homme, entre Jésus et le Christ, à l'intérieur de l'homme. Mais qu'est-ce qu'une frontière ? Une limite, une cassure, une coupure ? L'étymologie du mot la désigne à la fois comme ligne et espace imaginaire. Tillich a été un penseur en exil et a connu deux pays aux frontières particulières. La réception de sa pensée s'est agrandie pour traverser les frontières disciplinaires, notamment en philosophie et en sciences sociales. Son élaboration de la fragilité pathologique, mais aussi sa dynamique de la foi et sa conception du courage ont permis cette percée des frontières. Tillich semble appartenir à la culture générale des chercheurs qui se demandent 
comment résister aux graves traumas de la vie et comment se reconstruire, selon une expérience qu'il a lui-même vécue. On parle de la pathologie «borderline » par exemple. Les expériences de fragilité traumatique sont souvent des expériences de l'effraction de frontières personnelles, corporelles, psychiques, religieuses, qu'elles soient communautaires ou individuelles. Le thème de la frontière apparaît à la fois dans ses écrits systématiques et dans ses ouvrages biographiques. Tillich a éprouvé un choc lors de la première guerre mondiale et a éprouvé ce que l'on nomme désormais un post disaster trauma. Ce terme apparu discrètement aux États-Unis pour désigner les troubles des soldats revenus de la guerre du Vietnam a été étendu à toutes les suites traumatiques de choc, qu'il s'agisse du terrorisme, de la déportation. Le terme de choc est récurrent chez Tillich, à la fois comme thème biographique, et dans ses propos méthodologiques.

\section{La frontière dans les écrits autobiographiques}

Tillich a souvent présenté dans ses écrits biographiques deux chocs existentiels qui ont constitué des tournants dans son existence et sa pensée : la première guerre mondiale et l'exil en 1933. Dans les deux expériences, la question des frontières est centrale. Dans le cas de la guerre la remise en cause des frontières provoque le conflit armé, tandis que dans l'exil, le franchissement des frontières entraîne un changement radical de vie. Toutefois, ce changement ne s'est pas inscrit forcément tout de suite dans ses écrits. Le changement prend du temps. La frontière est un leitmotiv biographique et conceptuel tillichien :

Lorsque je fus prié de présenter l'évolution de ma pensée en fonction de ma vie, j’ai découvert à quel point le concept de "frontière" était un symbole de toute mon évolution personnelle et spirituelle. Mon destin m'a offert, presqu'en tout domaine, le choix entre deux possibilités. Mais comme je ne me sentais "chez moi" en aucune des deux, je n'ai jamais pu me décider l'une contre l'autre. (TILLICH, 1970, p. 7).

La première guerre mondiale est considérée comme l'un des tournants de sa vie. En même temps que la violence qui opère toutes sortes de ruptures, Tillich prend conscience des frontières sociales pendant la première guerre mondiale, pendant son travail d'aumônier militaire volontaire.

L'exil est le second tournant, mais n'empêche pas le maintien du lien avec son ancien monde. L'université qui l'accueille lui apparaît comme un 
refuge et un pont. Ses relations avec les psychologues, avec d'autres réfugiés, sont essentielles à son intégration au pays, comme à sa pensée. Il garde des liens avec l'ancien monde, et l'on peut remarquer que parfois ces liens se croisent, car une partie des psychanalystes viennent d'Europe, et sont euxmêmes en situation d'exil. Tillich note ainsi une difficulté de l'exil, suscitée par le risque de demeurer dans le cercle du passé. Son intégration sera renforcée par sa mise au service d'autrui, tandis qu'il préside le mouvement d'auto-assistance des émigrés d'Europe centrale : "Cette activité me mit en contact avec beaucoup de gens de l'ancien monde que je n'aurais jamais rencontrés autrement et me révéla la profondeur de l'angoisse et de la misère de l'homme ainsi que la grandeur, ordinairement cachée, du courage et du dévouement humain »(TILLICH, 1971, p. 51).

\section{La pensée-frontière}

Si la frontière est lieu où s'expérimente la fragilité, elle devient aussi un lieu envisagé positivement dans la pensée comme lieu d'apprentissage, aussi inconfortable fût- il : "En fait Tillich rappelle dans son esquisse autobiographique que la frontière est l'endroit idéal où acquérir des connaissances » (TILLICH, 1968, p. 51). La frontière développe l'ouverture, comme le rappelle André Gounelle : «Ainsi la frontière apparaît à Tillich non pas comme une ligne de démarcation qui isole et ferme, mais comme un lieu d'ouverture et de passage où s'opère une circulation et où se développent des échanges : là on rencontre l'autre, on entre en contact et on noue des relations avec lui »(GOUNELLE, 1992, p. 54)). La frontière se retrouve dans des textes autobiographiques, Aux frontières, en 1936, dans des interrogations théologiques en guise de titre d'ouvrage, Aux frontières de la science et de la morale (TILLICH, 1970) et comme métaphore de la pensée tillichienne qualifiée de "pensée frontière". Dans son ouvrage autobiographique de 1936, Aux confins, Tillich évoque la place de l'imagination dans sa pensée, avec ses richesses et ses ambiguïtés, dans un paragraphe intitulé, Entre la réalité et l'imaginaire. On voit ainsi un penseur ayant perdu sa mère jeune, s'efforcer de relire avec distance ses mécanismes de protection et leur répercussion sur sa philosophie et sa théologie :

De quatorze à dix-sept ans, je me retirais le plus souvent possible dans des mondes imaginaires qui me semblaient plus vrais que le monde extérieur. Avec le temps, cette imagination romantique s'est transformée en imagination philosophique. [...] Ce fut bon dans la mesure où cela me rendit apte à associer 
les catégories, à percevoir les abstractions en termes concrets (je dirais presque en couleurs), et à faire l'expérience d'une bonne série de possibilités conceptuelles .(TILLICH, 1971, p. 37).

Paul Tillich voit ainsi le passage de l'imagination romantique à l'imagination philosophique comme source d'association et de conceptualisation dont il fait l'expérience. Mais il en voit aussi les limites et les dangers car « une telle aptitude à l'imagination court le risque de prendre les créations de l'imagination pour des réalités, en négligeant l'expérience et la critique rationnelle, en pensant en monologues plutôt qu'en dialogues et en s'isolant de la coopération de l'effort scientifique »('TILLICH, 1971, p. 37-38).

\section{La frontière comme métaphore filée Dans les écrits systématiques}

Dans son œuvre systématique, Paul Tillich n'hésite pas à filer la métaphore de la frontière, comme en poésie. Il développe un ensemble métaphorique déclinant cette image sous les formes variées des frontières géographiques : fossé, muraille, place-forte... Tillich utilise la métaphore des murailles pour parler de la finitude, chez Nicolas de Cues et Kant, qu'elles soient temporelles, spatiales, causales ou substantielles. Ces murailles, qui nous sont données avec notre condition d'être humain, nous empêchent à tout jamais d'accéder à l'origine, à l'éternité. « Être fini veut dire être temporel. La raison ne peut pas percer les murailles de la temporalité pour atteindre la cause première, l'espace absolu, la substance universelle » (TILLICH, 2000, p. 118). Etre fini, c'est accepter les frontières de nos limites humaines. Mais en même temps, pour Tillich, il y a un franchissement : "Dans la vie concrète de la raison, il y a à la fois, union et désunion des forces essentielles et existentielles; des forces de création et de destruction » (TILLICH, 2000, p. 119) La raison est traversée par les frontières intérieur-extérieur. Historiquement, la raison autonome se libère et se maintient dans une lutte incessante contre l'hétéronomie. L'hétéronomie impose une loi (nomos) étrangère (hétéros) à l'une ou à l'ensemble des fonctions de la raison. "Du dehors » elle donne des ordres sur la manière dont la raison devrait saisir et façonner la réalité. Mais ce «dehors » ne se trouve pas seulement au dehors. Il représente en même temps un élément intérieur à la raison elle- même, la profondeur de la raison, ce qui rend dangereuse et tragique la lutte entre 
l'autonomie et l'hétéronomie. Il s'agit finalement d'un conflit à l'intérieur de la raison elle- même. » (TILLICH, 2000, p. 121). Dans le tome IV de la Théologie systématique, Tillich propose une autre issue que la frontière psychologique et l'esprit : l'image du saut kierkegaardien serait appliquée à chaque acte spirituel. «Au lieu de séparer l'esprit du domaine psychologique qui le conditionne, nous allons essayer de décrire la naissance d'un acte de l'esprit à partir d'une constellation de facteurs psychiques. Tout acte de l'esprit présuppose un matériel psychique donné et en même temps constitue un saut seulement possible par un soi complètement centré, autrement dit par un soi libre» (TILLICH. 1991, 31). Tillich utilise la métaphore du paravent d'images dans un chapitre sur l'ambiguïté des transformations personnelles. Dans le désir de rencontrer l'autre, nous vivons l'alternance du repli sur soi et de l'abandon à autrui. Nous projetons des images sur lui, ce qui nuit à la participation : «Dans la tentative de connaître l'autre, le repli sur soi s'exprime par une projection d'images de l'autre qui travestissent son être réel et ne sont que les projections de celui qui tente de connaître. Ce paravent d'images entre les personnes rend profondément ambigu toute connaissance de personne à personne (comme la montre amplement, par exemple, l'analyse de l'image des parents chez les enfants" (TILLICH, 1991, p. 86-87). Tillich distingue encore une autre forme de frontière, s'apparentant à une place forte. La tour d'ivoire mentale est une métaphore qui décrit la fragilité pathologique où s'est enfermé le sujet pour se protéger. Mais la frontière entre angoisse pathologique et existentielle n'est pas facile à dresser. Toutefois, il est possible de proposer des formes de guérison, sous l'impact de l' Etre Nouveau, capable de franchir certaines frontières et de guérir un soi disloqué. On peut supposer en voyant Paul Tillich déployer les frontières sur le plan discursif et métaphorique qu'il opère par la pensée, sous toutes ses formes, une intégration de ses expériences en une reconfiguration qui entremêle expériences, Ecritures, pensée conceptuelle en une ébauche d'une théologie poétique.

\section{Le thème de la captivité chez Paul Ricoeur}

Deux épreuves qui disloquent le soi, la captivité et la perte de son fils sont deux événements qualifiés de vendredi ou de samedi saint. Epreuves radicales où la fragilité s'incarne ? Paul Ricœur différencie la métaphore du poète et celle du philosophe : «Les métaphores du philosophe peuvent bien ressembler à celles du poète, en ce qu'elles opèrent comme ces dernières un 
écart par rapport au monde des objets et du langage ordinaire; mais elles ne se confondent pas avec les métaphores du poète » (RICOEUR, 1975, p. 395). Pour lui, « penser n’est pas poétiser »( RICOEUR, 1975, p. 395). Il nous semble cependant qu'il y a un croisement entre les deux exercices de pensée. Il existe un lien pensée-existence : Les chocs des deux guerres. Pour Paul Ricœur, tout événement historique marquant imprime sa trace chez le penseur. Son père fut tué lors de la première guerre $\mathrm{m}$ mondiale. Dans ses récits biographiques, il répétera mettre à distance sa propre expérience d'un camp de prisonnier et la vie dans les camps de concentration et la vie des prisonniers russes. Mais il rapporte avoir assisté à la violence de ce qui était subi par les soldats russes et d'autre part il a assisté à la sortie d'un camp de concentration après une longue marche forcée, dont il dit, pudiquement, qu'à un moment donné, il pensa ne pouvoir y survivre.

\section{La métaphore du Pays occupé}

Mais avant de «liquider » l'expérience de la captivité, Paul Ricœur a adossé sa réflexion sur la culpabilité aux symboles de la captivité et de l'infection. La captivité, symbole communautaire dans la Bible est commun à toutes les cultures et se personnalise ensuite. On le trouve chez Paul et Platon. Ainsi « la culpabilité la plus intériorisée peut récapituler tout le symbolisme antérieur, y compris celui de la souillure ; elle le reprend à son compte à travers le symbole de la captivité » RICOEUR, 1949, p. 304). Cette faute est un «paradis perdu » mythique qui ne détruit pas les structures fondamentales de l'homme: "le volontaire et l'involontaire tombent tels qu'ils sont en eux-mêmes au pouvoir du Rien, comme un pays occupé livré intact à l'ennemi» (RICOEUR, 1949, p. 8). Le thème du pays occupé en lien avec le mal ressurgit dans la réflexion du second tome de la Philosophie de la volonté. Pour clore le chapitre sur le serf-arbitre, Paul Ricœur parle de la surimpression existentielle de la bonté et du mal. Le mal n'est pas le contraire du bon, mais c'est plutôt un enlaidissement. "Le mal n'est pas le symétrique du bien, la méchanceté, le substitut de la bonté de l'homme, mais la flétrissure, l'obscurcissement, l'enlaidissement d'une innocence, d'une lumière et d'une beauté qui demeure »(RICOEUR, 1960, p. 306). Cette idée est présente dans le symbole de captivité que Ricœur met en parallèle avec l'état d'un pays occupé : «Car quand un pays tombe intact aux mains de l'ennemi, il continue de travailler, de produire, de créer, d'exister, mais pour l'ennemi ; il est responsable, mais son œuvre est aliénée ; cette superposition de l'esclavage 
à la disposition de soi, telle qu'un pays occupé peut en faire l'expérience, suggère l'idée semblable d'une superposition existentielle du mal radical et de la bonté originaire »(RICOEUR, 1960, p. 306). Cette réflexion parvient au terme d'une étude sur la captivité à Babylone, mais on peut entendre aussi des échos à la situation traversée. Ainsi entrent aussi en résonance, la réflexion sur le mal par contamination. Comment un être vivant une captivité peut-il s'extraire du mal subi : précisément en une double opération qui à la fois le dénonce et s'en distancie, mais non sans en avoir affirmé le caractère « d'infection ». Le mal n'est pas un néant mais une puissance des ténèbres qui vient de l'extériorité.

Infecter n'est pas détruire, ternir n'est pas ruiner. Le symbole pointe ici vers le rapport du mal radical à l'être même de l'homme, à la destination originaire de l'homme ; il veut dire que le mal, aussi positif, aussi séduisant, aussi affectant et infectant soit-il, ne saurait faire de l'homme autre chose qu'un homme ; l'infection ne saurait être une défection, au sens où les dispositions et les fonctions qui font l'humanité de l'homme seraient défaites, décréées au point de produire une autre réalité que la réalité humaine (RICOEUR, 1960, p. 305).

\section{La captivité dans la littérature}

Tout se passe comme s'il avait cherché à penser, et ainsi mettre à distance l'expérience de la souillure propre aux camps, expérience que l’on retrouve écrite sous forme de fiction, témoignage dans les récits des deux auteurs qu'il cite dans son ouvrage posthume : sur le deuil et l'agonisant, mais aussi dans les textes qu'il étudie dans un article de 1951¹, comme celui de Robert Antelme, L'Espèce humaine ou Margarethe Buber-Neuman Déportée en Sibérie. Ricoeur voit trois dimensions de l'homme dans cette littérature : "L'homme comme victime, l'homme comme bourreau, l'homme tentant de rompre le cercle infernal victime-bourreau «(RICOEUR, 1951, p. 150).

Les livres d'après-guerre que nous interrogeons sont apparentés par le ton : à travers de situations qui non seulement produisent la souffrance, mais paraissent devoir ruiner tout espoir et enfermer l'homme sans issue dans une conscience de destruction, on peut alors se demander si, à travers ces

Robert Antelme, L'Espèce humaine, Margarethe Buber-Neuman Déportée en Sibérie, Jean Cayrol, Je vivrai l'amour des autres et Lazare parmi nous, Gheorgin Constantin, La vingt-cinquième heure, E. Kogan, SS. Stadt et L'enfer organisé,, Th. Plievier, Stalingrad, F.R. Stuart, La Colonne de feu, D. Rousset, Les jours de notre mort. 
situations, ne nous est pas révélé quelque chose d'essentiel concernant l'homme - quelque chose que la banalité dissipe, quelque chose peut-être que le bonheur masque et que Job a bien connu » (RICOEUR, 1951, p. 150).

Dans cette littérature, émerge une souffrance qui s'apparente à la souffrance du Vendredi Saint. "Il y a une connaissance par la pitié » (RICOEUR, 1951, p.149). Ce qui s'exprime, c'est « la connaissance du Samedi Saint (RICOEUR, 1951,150)» L'autobiographie écrite ou orale offre des niveaux de strates de recomposition multiples. Il y a un transfert possible du courage de la victime du passé à la victime à venir. "Toute grande littérature est littérature de l'extrême » (RICOEUR, 1951, ) ${ }^{1242}$.

\section{L'imaginaire des camps et de la mort}

Paul Ricoeur évoque les situations de catastrophes, comme les pandémies, en lien avec sa réflexion sur les camps de prisonniers. Il en parle dans la Philosophie de la volonté rédigée pendant la seconde guerre mondiale et reprendra ce terme de situation-catastrophe dans l'ouvrage posthume pour en 2008. Dans le premier tome de la Philosophie de la volonté, il parle de la tendance agressive de la vie et interroge le lien entre la situation catastrophe et l'amitié dans un paragraphe sur la confusion affective :

Dans telle situation extrême n'ai-je pas à choisir entre d'une part ma vie et d'autre part, la vérité, mes amis, ma foi ? Précisément si à certains moments ma vie m’apparait rassemblée en une valeur globale, ce n'est pas de l'intérieur de l'expérience affective indéfinie et bariolée, mais de l'extérieur, à partir de la mort. C'est la mort qui donne son unité à la vie, en ce sens que seule une situation de catastrophe, en m'acculant à choisir entre ma vie et celle de mes amis, a le pouvoir de mettre globalement en question, mon existence (RICOEUR, 1948, p. 11) .

Ie terme de » danger de mort » en allemand reçoit toute la simplicité dont il est capable : face à lui, "l'être en vie » apparait alors comme une situation totale qui a la simplicité, sinon d'un acte que je pose, du moins d'un état qui est l'état même d'exister corporellement » (RICOEUR, 1948, p.11).

Dans l'ouvrage posthume de Paul Ricœur, la référence à Georges Semprun surgit dans le paragraphe sur les « figures de l'imaginaire de la mort » tandis qu'il réfléchit sur la dimension éthique de l'accompagnement comme « capacité à accompagner en imagination et en sympathie la lutte de l'agonisant encore vivant, vivant jusqu'à la mort » (RICOEUR, 2007, p. 48). 
«Il me vient ici l'évocation d'un autre témoignage, celui de Jorge Semprun dans l'Ecriture ou la vie ».(SEMPRUN, 1994). Jorge Semprun accompagne les derniers moments de Maurice Halbwachs. Ricoeur parle de 1 'imaginaire de la mort et de Primo Levi :

Oui, j’aurais tendance à croire que la mort personnifiée, agissante et destructrice, surgit dans l'imaginaire au point où les morts déjà morts et les moribonds qui vont être morts deviennent eux-mêmes indistincts ; C'est le cas dans les grandes épidémies -de peste, de choléra,..- et ce fut celui dans les camps de concentration, dans cette situation extrême où le survivant provisoire est environné, cerné, submergé par la masse indistinct des morts et des moribonds et habité par le sentiment de la très grande probabilité de sa mort prochaine, de l'imminence de cette mort. Alors, il s'imagine, il se perçoit comme faisant déjà partie de cette masse indistincte des morts et des moribonds. J'insiste sur l'effet de masse et l'effet d'indistinction. Il n'est effectif que dans les situations limites que j’ai dites : épidémie, extermination (RICOEUR, 2007, p. 53-54).

\section{Un autre vendredi saint de son existence}

Paul Ricœur conjugue les différents modes de pensée pour conjurer l'expérience de la souffrance et du mal, comme il le fera face à un autre vendredi saint de sa vie. une réflexion de Paul Ricœur extraite de Réflexion faite :

Peu de jours après [...] S'abattit le coup de foudre qui lézarda notre vie entière : le suicide de notre quatrième fils [...] Je trouvai quelque secours dans un essai que j'avais écrit à l'automne précédent et dont la publication survint peu après la catastrophe. Dans ce texte intitulé Le mal. Un défi à la philosophie et à la théologie, je tentais de formuler les apories suscitées par le mal-souffrance et occultées par les théodicées. Mais aussi, j’y esquissai pour finir les étapes d'un cheminement de consentement et de sagesse. Je me découvris soudain le destinataire imprévu de cette âpre méditation (RICOEUR, 1995, p. 79-80).

Face au mal, l'homme s'interroge : Pourquoi ? Pourquoi moi ? Son cri se transforme en parole, puis en écriture et lecture. Il part à la recherche d'écrits qui expriment ce qu'il ressent. Les psaumes bibliques de plainte expriment précisément de manière universelle la douleur du mal et les sentiments de détresse. Ce sont des poèmes qui ont traversé les siècles et s'insèrent dans les pratiques de prières, seul ou en communauté, lors du culte, et de l'enterrement. "Il a fallu d'abord que la parole élève à l'expression articulée ce qui aurait pu n'être que cris, larmes et 
gémissements. Il a fallu encore que l'écriture - et l'écriture rythmée selon les canons de la poétique hébraïque- élève la parole au rang de texte susceptible d'être mémorisé, récité, chanté » (RICCEUR, 1998, p. 292). Ce psaume Inde intègre le cri "Pourquoi m'as-tu abandonné », qui sera repris par le Christ sur la croix. Ce Psaume passe de la plainte à la louange, comme dans de nombreux psaumes, mais dans un mixte des deux genres. Cette écriture de la souffrance se construit par un travail d'effacement et de désingularisation : La désingularisation consiste à changer la personne verbale, à procéder à une distanciation qui permet d'englober le lecteur dans le moment initié par le texte.Il y a un retournement de la plainte en louange. Le chemin du Psaume invite le lecteur à en faire autant et à rejoindre ce nouveau paradigme, par désingularisation de la souffrance. Pour Ricœur, la force du cri du Christ, «Pourquoi m'as-tu abandonné? »est qu'il n'est pas juste une citation, mais le Christ vient «habiter» le cri : Jésus mourant revêt sa souffrance dans les mots du Psaume, qu'il Habite ainsi de l'intérieur » (RICOEUR, 1998, p. 287). C'est un poème qui élève «au rang de la parole, de l'écriture, enfin du texte, des moments fondamentaux de l'expérience religieuse » (RICOEUR, 1998, p. 288). Ces expériences religieuses peuvent être : «Une confiance sans réserve à l'égard de Dieu [...] le sentiment de dépendance absolu [...] la conscience d'appartenir à une économie du don qui précède tout mouvement humain de charité [...] l'élan de l'Ultimate concern » (RICOEUR, 1998, p. 288). Ricœur reprend ici le terme de Tillich, Ultimate concern. La prière s'adresse à un Toi caché toi et témoigne d'un être-avec-Dieu-malgré et débouche sur un appel à la compassion :

Une dernière condition à satisfaire par le suppliant d'aujourd'hui serait peut-être qu'il découvre une secrète parenté entre ce qu'on pourrait appeler souffrance de Dieu [...] Et l'appel à une pratique personnelle et communautaire de la compassion à l'égard des frères humains souvent plus souffrants que coupables (RICOEUR, 1998, p. 313).

\section{Articulations faille-Ecritures}

Dans les Fragments, Paul Ricœur rassemble les deux expériences du vendredi ou samedi saint dans le commentaire d'un ouvrage de Georges Semprun. Face au mal, au suicide il y le choix entre vie et écriture : Tout le ne craignezpas est ici en négatif : «la faille au cœur de toute existence » (RICOEUR, 2007, p. 68). 
L'alternative de Jorge Semprun : seul un suicide pourrait signer, mettre fin volontairement à ce travail de deuil inachevé (mis en demeure de " choisir» entre l'écriture et la vie. N'est-ce pas -soit dit en passant- une autre figure de la mort : le suicide. Peut-être fallait-il d'abord choisir la vie contre pour pouvoir un jour écrire et vivre. Passage de tous, de moi aussi par l'aphasie ? Mais n'est-ce pas de cet état que Je sors en souscrivant ces pages ? "Deuil de l'Ecriture » en vue du deuil de la mémoire ? Car nous ne sommes pas forts. Il faut plier un peu, longtemps, avant d'affronter l'orage. Car c'est aussi son suicide qu'il me faut accepter. Ici me touche la question de Jorge Semprun : « suis-je vraiment revenu (RICOEUR, 2007, p. 68)?

Dans cet ouvrage posthume, un retournement s'opère chez Ricœur : une réponse nait, dans sa méditation sur les versets de Jean : «Qui veut sauvegarder son existence la perdra. Qui aura perdu son existence la sauvegardera » (Jean 12, 25). Ce paradoxe johannique doit être pensé dans sa verticalité. Sauvegarder peut signifier soit s'accrocher à la vie, soit se dessaisir. Ricœur propose la corrélation « détachement de soi » et «transfert sur l'autre du détachement » (RICOEUR, 2007, p. 87 ). «Le Fils de l'Homme est venu non pour être servi mais pour servir. Le lien mort-survie dans l'autre est noué dans le service pour... associé au don de la vie» (RICOEUR, 2007, p. 91). A son tour, le penseur endosse la posture christique « Je remets mon esprit à Dieu pour les autres. Ce lien, cette transmission à son sens au-delà de moi et un sens y est caché auquel Dieu peut-être m'associera d'une façon que je ne peux imaginer » RICOEUR, 2007, p. 130). Un mot me revient à nouveau : « l'espérance qu'elle me survive. »(RICOEUR, 2007, p. 68).

\section{Expérience existentielle et transcendance}

Comment articuler expérience existentielle et transcendance ? La philosophie de la religion s'insère dans le cercle herméneutique entre la question existentielle et la réponse théologique. Nous voyons les thèmes qui ont fait choc dans l'expérience des auteurs, Paul Tillich et Paul Ricoeur traverser leur oeuvre, au fil du temps et se recomposer selon le type de textes, autobiographiques, conceptuels, littéraires, exégétiques, systématiques. Cette reconfiguration permet une distanciation de l'épreuve, puis une réappropriation. Ainsi chez Paul Tillich, la figure de la frontière est une métaphore autobiographique, une figure de pensée (la création comme frontière franchie), une méthode théologique. Ces figures traversent un cercle herméneutique qui circule entre écriture de soi, écriture philosophique, 
écriture théologiques et Ecritures. Chez Paul Ricoeur on voit un thème biblique de la croix reconfiguré et réinterprété. Le cercle herméneutique circule entre l'expérience des camps et pensée philosophique, systématique, exégèse et interprétation littéraire. Il n'y a pas de rupture entre pensée figurative et pensée conceptuelle. L'usage des métaphores montre la perméabilité de l'arc herméneutique. Dans l'existence, nos traversons des épreuves que nous subissons. Mais une force donnée par la transcendance nous permet de les traverser en les reinterprétant, en les reconfigurant à la lumière du Christ. La philosophie de la religion, qui survole les crêtes de la philosophie et de la théologie, met à jour ce travail de reconfiguration. Paul Tillich propose un processus de reconfiguration en trois temps : engagement, détachement et ressaisie existentielle. Ce chemin relie la profondeur de la vie et la foi. il y a un détachement par rapport à l'expérience, nécessaire à la pensée, suivi d 'une ressaisie existentielle des existentiaux ou situations-limites que sont l'angoisse, le non-être. Dans son analyse de la prédication, Raphaël Picon rappelle l'un des usages pastoraux des existentiaux : ils doivent être réappréhendés à l'occasion de l'interprétation des symboles et expressions que "Royaume de Dieu" :

La réinterprétation par Tillich de ces données va toujours dans le sens de leur ressaisie existentielle. Chacun de ces éléments est compris de manière à susciter une prédication structurée par une rhétorique de l'affrontement par l'affirmation. Affrontée au non-sens, à la finitude, à la culpabilité, au mépris de soi, à la répression morale, on réagit par l'énoncé aux valeurs performatives d'une puissance d'être, d'une "Présence spirituelle" et créatrice qui permet de surmonter le non-être (PICON, 2007,170).

La métaphorisation et la désingularisation sont des opérations herméneutiques qui permettent de relier la pensée et l'ultime.

\section{Bibliographie}

ANTELME, Robert, L'Espèce humaine, Paris, Gallimard, 1978

BÉLAND, Jean-Pierre, Finitude essentielle et aliénation existentielle, BUBER-NEUMANN, Margarethe ,Déportée en Sibérie, Paris, Editions du Seuil

CAYROL, Jean, Je vivrai l'amour des autres, A l'Enseigne du Cheval Ailé, le Livre de Poche, 1972 
CAYROL, Jean, Lazare parmi nous, Editions Léo Scheer, 2006

DOUGLAS, Nelson, (éds); Paul Tillich, prédicateur et théologien pratique,, Actes du XVIe colloque international Paul Tillich, Mont- pellier, 2005, Berlin, Lit, 2007.

FREY, Daniel, Lecture philosophique et lecture théologique de La Bible chez Paul Ricœur, http://RICGEUR.pitt.edu/ ojs/index.php/RICGEUR/article/viewFile/152/68. Consulté le 23 juin 2013.

FAESSLER, Marc, Le mal, la méditation philosophique de Ricœur à l'épreuve du tragique, Laval théologique et philosophique 65:/3 (2009), p. 431-450.

JASPERS, Karl, Introduction à la philosophie, trad.Jeanne HERSCH, Paris, 10/18, 2001

JASPERS, Karl, Psychologie der Weltanschauungen, Berlin/ Göttingen/Heidelberg, Springer-Verlag, 1960 (1954)

GOUNELLE, André, "La notion de frontière à partir de Paul Tillich », Autres temps. Les cahiers du Christianisme social Constantin Virgil GHEORGHIN, La vingtcinquième heure, Paris, Pocket, 2006.

E. KOGON, SS. Stadt, Frankfurt am Main, Europäisch Verlagsanstalt, 1946.

E. KOGON, L’enfer organisé, Le système des camps de concentration, Paris, La jeune Parque 1947. KOGON, Les jours de notre mort, Paris, Editions du Pavois,1946

MILLER, Patrick D., Interpreting the Psalms, Minneapolis, Fortress Press,1986

Raphaël PICON, «Le ministère pastoral chez Paul Tillich », in Marc DUMAS, Mireille HEBERT,, Nelson DOU- GLAS (eéds); Paul Tillich, prédicateur et théologien pratique,, Actes du XVIe colloque international Paul Tillich, Montpellier, 2005, Berlin, Lit, 2007

«PLIEVER, Thierry, Stalingrad, Edition Marin,Réédition Cologne,Parkland-Verlag, 2004

RICOEUR, Paul, pHilosophie de la volonté, t. 1, Le Volontaire et l'involontaire, Aubier, 1949

RICOEUR, Paul, pHilosophie de la volonté, t. 2, Finitude et culpabilité, Aubier, 1960

RICCEUR, Paul, «Connaissance de l'homme par la littérature de malheur », Foi Education 15 (1951), p. 149-156

Paul RICEUR, La Bible et 1 imagination, Fonds Ricœur.

RICCEUR, Paul, «Introduction », in Jocelyn DIMPLY, Paul Tillich et le symbole religieux, Préface de Paul Ricœur, Encyclopédie Universitaire, Paris, Jean-Pierre Delarge Editeur, 1977 
Paul RICEUR, La Métaphore vive

Paul RICEUR, Paul, Le défi du mal. Un défi à la philosophie et à la théologie, Genève, Labor et Fides, 1986

RICCeUr, Paul, LACOCQUE, André, Penser la Bible, Paris, Editions du Seuil, 1998

RICCEUR, Paul, Vivant jusqu'à la mort suivi de Fragments, Paris, Editions du Seuil, coll. «L'ordre philosophique », 2007

RICCEUR, Paul, Cinq Etudes Herméneutiques, Genève, Labor et Fides, 2013

RICCEUR, Paul, La critique et la conviction. Entretien avec François Azouvi et Marc de Launay, Paris, Hachette Littérature, 2002

RICCEUR, Paul, Réflexion faite : Autobiographie intellectuelle. Paris, Esprit, 1995

RICOEUR, Paul, Ecrits et Conférences 2. Herméneutique, Daniel Frey, Nicola Stricker (Eds), Editions du Seuil, coll. Couleurs Idées, Paris, 2010

RICCEUR, Paul, Lectures 3,

RICCEUR, Paul, «Le scandale du mal», Esprit, juillet (2005)

Joël ROMAN, Joël, TASSIN, Etienne, "Paul RICCEUR J'attends la renaissance », in J. MESSAGE, J.ROMAN, E. TASSIN, À quoi pensent les philosophes? Paris, coll « Autrement $» 102,1988$.

RICCEUR, Paul, «Herméneutique philosophique et herméneutique biblique », in François Bovon, Grégoire Rouillé (eds), Exegesis, Neuchâtel/paris, Delachaux et Nietslé, coll. "Bibliothèque théologique », 1976, p. 216-228

RICCEUR, Paul, «Connaissance de l'homme par la littérature de malheur », Foi Education 15 (1951) p.149-156

ROUSSET, David, Les jours de notre mort, Paris, Éditions du Pavois, 1947

SEMPRUN, Jorge, L'Écriture ou la vie, Souvenirs, Paris, Gallimard, 1994

SAINT-CHERON, (DE) Mickaël, Du Juste au Saint . Ricœur, Rosensweig, Levinas, Desclée de Brower, 2013

STUART, La Colonne de feu, Paris, Editions du Temps présent,1949

TILLICH, Paul, Réflexions autobiographiques, suivi de Le Christianisme et les religions, Fernand Chapey (trad.), Paris, Aubier/Montaigne, coll. « La Philosophie en poche »,1968 


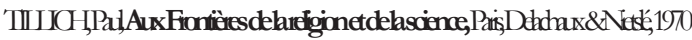

TILLICH, Paul, Aux confins, Esquisse autobiographique, Paris, Planète, coll. «L'expérience intérieure », 1971.

TILLICH, Paul, Théologie Systématique, Raison et révélation, vol. I, trad. André Gounelle, Mireille Hébert, Claude Conedera, Paris/Genève/Laval, Cerf/Labor et Fides/Presses de l)Université Laval, 2000.

TILLICH, Paul, Théologie Systématique, La Vie et l'Esprit, vol. IV, trad. Jean-Marc Saint, Paris/Genève/Laval, Cerf/Labor et Fides/Presses de l’Université Laval, 1991.

TILLICH, Paul, Religion biblique et ontologie, Paris, Presses Universitaires de France, 1960 TILLICH, Paul, Le Courage d' Être, trad. Fernand Chapey, Paris, Casterman, 1967

TILLICH, Paul, Documents biographiques, Paris/Genève/Laval, Cerf/Labor et Fides/ Presses de l'université Laval, 2002

TILLICH, Paul, L' Être Nouveau, trad. Jean-Marc Saint, Paris, Planète,1969

TILLICH, Paul, Existential philosophy, in Philosophical Writings, Berlin/New York , Gunther/de Gruyter, 1989 